COMMUNICATIONSARISING

Electron-spin domains

\section{Magnetic enhancement of superconductivity}

1 $\mathrm{n}$ inhomogeneous superconducting state, not yet conclusively identified, was predicted by Fulde and Ferrell ${ }^{1}$ and Larkin and Ovchinnikov ${ }^{2}$ (FFLO) to arise in superconductors with strong Pauli limiting, a consequence of the electrons' Zeeman (spin) energy in a magnetic field. Radovan et al. ${ }^{3}$ propose that the observed cascades of steps in magnetization of the heavy fermion superconductor $\mathrm{CeCoIn}_{5}$, within the recently discovered second low-temperature state ${ }^{3,4}$, are due to transitions between Landau-level (LL) states with different $m$-quanta vortices, expected under certain conditions when the magnetic field is swept within the FFLO state $^{5-7}$. The authors then conclude that the observed steps in magnetization constitute a proof that the low-temperature state in $\mathrm{CeCoIn}_{5}$ is indeed an FFLO state. We argue that this interpretation of the observed steps in magnetization cannot be supported on either quantitative or qualitative grounds.

The relative effectiveness of the orbital ${ }^{8}$ and paramagnetic ${ }^{9}$ (Pauli) limiting effects in suppressing superconductivity is reflected in the Maki parameter $\alpha=\sqrt{2 \mathrm{H}_{\mathrm{c} 2}{ }^{0} / H_{\mathrm{P}}}$. A large $\alpha$ indicates a small Pauli limiting field, $H_{\mathrm{P}}$ (stronger Pauli limiting), and/or a large orbital limiting field, $H_{\mathrm{c} 2}{ }^{0}$. Radovan et al. ${ }^{3}$ obtain $\alpha \approx 13$ by using $H_{\mathrm{p}} \approx 4 \mathrm{~T}$, which assumes an electron $g$-factor of 2 for $\mathrm{CeCoIn}_{5}$. But the experimental value of the superconducting critical field is $H_{\mathrm{c} 2}=12 \mathrm{~T}$, three times the Pauli (upper) limit used by Radovan et al. Therefore, $H_{\mathrm{P}}=4 \mathrm{~T}$ is unphysical.

$H_{\mathrm{p}}$ can be estimated both theoretically and experimentally. It has been estimated by fitting the critical field $H_{\mathrm{c} 2}$ of $\mathrm{CeCoIn}_{5}$ to a model for a $d$-wave superconductor with an FFLO state ${ }^{10}$ to give an electron $g$-factor of 0.64 or $H_{\mathrm{P}}=12.8 \mathrm{~T}$. A very conservative lower bound of $H_{\mathrm{P}}>10 \mathrm{~T}$ can be made simply by noting that $H_{\mathrm{c} 2}=10 \mathrm{~T}$ at $1 \mathrm{~K}$, where an FFLO state cannot influence $H_{\mathrm{c} 2}$.

Theoretically, the Maki parameter must exceed 9 for an $m \neq 0$ LL state to become a ground state ${ }^{5} . H_{\mathrm{p}}=12.8 \mathrm{~T}$ gives $\alpha \approx 4.5$, which is a third of the value derived by Radovan et al., and half the minimum required for observation of $m \neq 0$ LL superconducting states. The number of observed steps (tens) also seems to be orders of magnitude greater than that expected theoretically ${ }^{5}$ for a material with $\alpha$ close to that of CeCoIn ${ }_{5}$. Only one non-zero state $(m=1)$ is expected $^{5}$ for $\alpha \geqslant 9$, and two such states $(m=1,2)$ appear $^{5}$ at $\alpha \geqslant 20$, far greater than $\alpha \approx 4.5$ for $\mathrm{CeCoIn}_{5}$.

In addition, the number of higher LL states is theoretically expected to increase as the applied field approaches the parallel orientation $(\theta \rightarrow 0)$, with $m \rightarrow \infty$ at $\theta=0$ (refs $6,7)$. This prediction opposes the trend shown by the results of Radovan et al. Thus, we believe that the observed steps in magnetization cannot be due to the higher LL states induced by the FFLO effects in $\mathrm{CeCoIn}_{5}$.

The heat-capacity data shown in Fig. 2 of Radovan et al. ${ }^{3}$ are inconsistent with our data ${ }^{4}$, and miss a large narrow peak associated with the first-order nature of the superconducting phase transition below $1 \mathrm{~K}$. A related point is that it is also incorrect to identify the order of the phase transition on the basis of the presence or absence of a temperature swing at a phase transition in the described magnetocaloric setup (inset to their Fig. 2). Such swings are expected both at first- and at second-order phase transitions ${ }^{11}$.

Roman Movshovich*, Andrea Bianchi $\dagger$, Cigdem Capan ${ }^{\star}$, Marcelo Jaime*,

\section{R. G. Goodrich $\neq$}

* Los Alamos National Laboratory, Los Alamos, New Mexico 87545, USA

e-mail:roman@lanl.gov

$\dagger$ Hochfeld Labor Dresden, Forschungszentrum

Rossendorf, Postfach 5101 19, 01314 Dresden,

Germany

$\$$ Department of Physics and Astronomy, Louisiana State University, Baton Rouge, Louisiana 70803-

\section{1, USA}

1. Fulde, P. \& Ferrell, R. A. Phys. Rev. 135, A550-A563 (1964).

2. Larkin, A. I. \& Ovchinnikov, Y. N. Zh. Eksp. Teor. Fiz. 47, 1136 (1964).

3. Radovan, H. et al. Nature 425, 51-55 (2003).

4. Bianchi, A. et al. Phys. Rev. Lett. 91, 187004 (2003).

5. Buzdin, A. I. \& Brison, J. P. Phys. Lett. A 218, 359-366 (1996).

6. Buzdin, A. I. \& Brison, J. P. Europhys. Lett. 35, 707-712 (1996).

7. Shimahara, H. \& Rainer, D. J. Phys. Soc. Jpn 66, 3591-3599 (1997)

8. Ginzburg, V. L. Sov. Phys. JETP 4, 153-160 (1957).

9. Clogston, M. Phys. Rev. Lett. 9, 266-267 (1962).

10. Won, H. et al. Preprint at <http://xxx.lanl.gov/cond-mat/ 0306548> (2003).

11. Jaime, M. et al. Phys. Rev. Lett. 89, 287201 (2002).

Radovan et al. reply - Movshovich et al. suggest that our calorimetric observation of the FFLO superconducting phase boundary ${ }^{1}$ and its angular dependence are inconsistent with their own data reporting the possible presence of an FFLO phase, and that we have incorrectly used magnetothermal measurements to identify the order of the FFLO transition. They also contest that the correlation of the magnetization steps with the calorimetrically identified FFLO superconducting phase of $\mathrm{CeCoIn}_{5}$ is coincidental. We consider each of these points in turn.

First, our calorimetrically determined FFLO phase boundary is in fact in good agreement with their own phase diagram ${ }^{2}$ for HII [110] and with their earlier calorimetric data ${ }^{3}$ reporting the absence of an FFLO phase transition for $H \|[001$ ] down to $0.1 \mathrm{~K}$. In addition, our measurements of the variation of $C_{\mathrm{p}}$ with field - data used to determine the location of the FFLO and $H_{\mathrm{c} 2}$ superconducting phase boundaries - agree with new measurements ${ }^{4}$ at $0.5 \mathrm{~K}$ for $H 川[110]$ and with earlier work ${ }^{5}$ at $0.25 \mathrm{~K}$ and $0.8 \mathrm{~K}$ for
$H I I[001]$. Because our specific-heat measurements only extend down to $0.15 \mathrm{~K}$, we are unable to address the merits of their revised report ${ }^{2}$ of a possible FFLO phase transition at $0.13 \mathrm{~K}$ for $\mathrm{HI}[001]$.

Second, the determination of the secondorder nature of the FFLO phase boundary from the absence of a detectable heat pulse in the magnetothermal data presented in ref. 1 for $0.25 \mathrm{~K}$ is also in good agreement with the results in ref. 2. As the first-order nature of the $H_{\mathrm{c} 2}$ transition at that temperature has already been established ${ }^{3,6}$, the temperature changes at the transition can be identified as the release and absorption of latent heat. Although our determination of a change in the order of $H_{\mathrm{c} 2}$ at $T_{0}=1.25 \pm 0.15 \mathrm{~K}$ for $H I I[110]$ contradicts the originally inferred value $^{6}$ of $0.7 \mathrm{~K}$, it is in increasingly good agreement with more recent values ${ }^{2}$ of $1.0 \mathrm{~K}$ for $H \|[100]$ and $1.1 \mathrm{~K}$ for $H \|$ [110].

Third, decreasing the angle, $\theta$, between the applied field and the superconducting layers at constant external field reduces the value of the field component normal to the layers, $B_{\perp}$. This in turn decreases the Landau-level spacing and allows the order parameter to assume higher states, $m$, for a given FFLO wavevector, $Q=Q(B)$. In our case, however, the data were obtained by increasing the field at constant angle. If the total induction remained constant, the Landau index would then decrease in an increasing field. However, the FFLO wavevector also changes with each step, precluding prediction of the optimal Landau index at a given field at the present time.

A full treatment would require consideration of large effective masses, spin-orbit coupling, $g$-values ${ }^{7}$ and the spatial variation in the magnetic flux (that is, finite GinzburgLandau parameter, $\kappa$ ). To our knowledge, the first work implementing some of these effects is given in refs 8,9 , but $\mathrm{CeCoIn}_{5}$ displays a first-order rather than a second-order phase transition at the critical field, and it is not clear how this affects these predictions for the Landau-level quantization. We have already proposed a method of incorporating strong electron-electron coupling and many-body effects to account for the experimentally determined Pauli limit ${ }^{1}$.

H. A. Radovan ${ }^{\star}$, N. A. Fortune,

T. P. Murphy, S. T. Hannahs, E. C. Palm,

S. W. Tozer, D. Hall

${ }^{\star}$ NHMFL, Florida State University, Tallahassee,

Florida 32310, USA

e-mail: radovan@magnet.fsu.edu

1. Radovan, H. et al. Nature 425, 51-55 (2003)

2. Bianchi, A. et al. Phys. Rev. Lett. 91, 187004 (2003).

3. Bianchi, A. et al. Phys. Rev. Lett. 89, 137002 (2002).

4. Aoki, H. et al. J. Phys. Cond. Mat. 16, L13-L19 (2004).

5. Ikeda, S. et al. J. Phys. Soc. Jpn 70, 2248-2251 (2001).

6. Tayama, T. et al. Phys. Rev. B. 65, 180504 (2002).

7. Won, H. et al. Preprint at <http://xxx.lanl.gov/cond-mat/ 0306548> (2003).

8. Klein, U. Preprint at $<$ http://xxx.lanl.gov/cond-mat/0312095> (2003).

9. Klein, U., Rainer, D. \& Shimahara, H. J. Low Temp. Phys. 118, 91-104 (2002). 